\title{
TRANSTORNOS MENTAIS E COMPORTAMENTAIS RELACIONADOS AO TRABALHO: O QUE A PSICOLOGIA TEM A DIZER E A CONTRIBUIR PARA A SAÚDE DE QUEM TRABALHA?
} Wladimir Ferreira de Souza $\star$

\begin{abstract}
Resumo
Este breve ensaio tem por objetivo colocar em discussão algumas das contribuições advindas de importantes publicações a respeito da problemática relacionada aos transtornos mentais e comportamentais e suas vinculações com o trabalho. Quanto a isso, verifica-se que a Psicologia, enquanto ciência e profissão, encontra-se diante de inúmeros desafios a superar, os quais concernem tanto à formação dos profissionais, quanto à sua atuação nos mais diversos campos.

Palavras-chave: trabalho; transtornos mentais; Psicologia.

\section{Mental and BeHaVioral DisORders Related WORK:}

\section{WHAT PSYCHOLOGY HAS TO SAY AND CONTRIBUTE TO HEALTH OF PEOPLE WHO WORK?}

\begin{abstract}
This brief essay seeks to speak on the contributions of some important publications about issues related to mental and behavioral disorders and their linkages with the work. In this regard, it appears that psychology as a science and profession, is faced with many challenges to overcome, which concern both the training of professionals as to their performance in various fields.
\end{abstract}

Keywords: work; mental disorders; Psychology.

\footnotetext{
^Psicólogo. Doutor em Psicologia Social pelo Programa de Pós graduação em Psicologia Social da Universidade do Estado do Rio de Janeiro, tendo realizado formação complementar (Programa de Doutorado com Estágio no Exterior - PDEE/CAPES/MEC) na Université de Provence (França). Professor doutor, colaborador no curso de Pós graduação lato sensu em Psicologia do Trabalho e das Organizações da Universidade de Barra Mansa/RJ. Endereço: Prefeitura Municipal de Duque de Caxias, Secretaria Municipal de Saúde. Alameda Dona Esmeralda, nº 206 Praça Benzo de Kavour. Jardim Primavera. Duque de Caxias, RJ - Brasil. CEP: 25215-260.

E-mail: souzalandi@uol.com.br
} 


\section{INTRODUÇÃo}

Em nossa sociedade, o trabalho é mediador de integração social, seja por seu valor econômico (subsistência), seja pelo aspecto cultural (simbólico), tendo assim, importância fundamental na constituição da subjetividade, no modo de vida e, portanto, na saúde física e mental das pessoas.

No âmbito da ciência psicológica, o Conselho Federal de Psicologia (CFP), através do Centro de Referência Técnica em Psicologia e Políticas Públicas, elaborou documento (CENTRO DE REFERÊNCIA TECNICA EM PSICOLOGIA E POLÍTICAS PÚBLICAS, 2008) no qual assinala que o psicólogo, independente do lugar em que esteja atuando, poderá deparar-se com questões do processo saúde-doença em sua relação com o trabalho. Entende-se que os preceitos do campo da Saúde do Trabalhador repercutem na prática profissional psi, implicando numa releitura teórica e metodológica dessa prática, proporcionando um olhar sobre o ser humano na relação com a sua atividade, isto é, na forma pela qual se insere no processo produtivo, além das condições, da organização e da divisão do trabalho.

Independentemente do seu espaço de atuação e de quem o contrata, o psicólogo deve sempre colaborar - preferencialmente de forma interdisciplinar e em equipes multiprofissionais - com a luta pela vida e saúde dos humanos, inclusive em seu trabalhar.

O CFP orienta os psicólogos a sempre perguntarem sobre o trabalho no processo de investigação diagnóstica. Levantar aspectos da organização do trabalho, identificar as exigências físicas e mentais, inquirir sobre a percepção do trabalhador a respeito dos riscos, obter informações sobre o posto de trabalho, as condições ambientais e o processo de trabalho fornecem importantes elementos para melhor compreensão do quadro patológico. Contudo, ressalta-se que a ênfase na psicopatologia pode encobrir o contexto em que se dá a relação entre o trabalhador e seu trabalho. O que a Clínica do Trabalho em Psicologia (BENDASSOLLI; SOBOLL, 2011) tem como regra de ouro é que antes de mais nada o trabalho humano precisa ser cuidado, para além de um trabalhador específico, o que não exclui a eventual necessidade de atendimento psicológico individual. Segundo Jacques (apud CONSELHO FEDERAL DE PSICOLOGIA, 2010, p. 10-11), deve "ser levada em conta a história de vida e de trabalho da pessoa, o que ajuda a explicar como ela se relaciona com o trabalho e com a situação que ali ocorre e que causa sofrimento para uns e não para outros".

Verifica-se que sobretudo nas décadas de 1990 e 2000, as repercussões psíquicas do trabalho passam a ganhar espaço nos consultórios e também no âmbito acadêmico, científico e governamental, no Brasil. Esses agravos, que até então não identificados, evidenciam a complexidade que envolve a avaliação da relação entre o processo saúde/doença e o trabalho.

Conforme Seligmann-Silva et al. (2010a), as expressões clínicas dos transtornos mentais e comportamentais relacionados ao trabalho correspondem a vários diagnósticos presentes na Classificação Internacional de Doenças (CID-10) e são 
reconhecidos como relacionados ao trabalho tanto pelo Ministério da Saúde (Portaria $n^{\circ}$ 1339/GM, de 18 de novembro de 1999), como pelo Ministério da Previdência e Assistência Social (Decreto no 3048, de 1999) (BRASIL, 1999a, 1999b).

Conforme publicado pelo Ministério da Saúde (BRASIL, 2001), uma estimativa da Organização Mundial da Saúde constatou que os transtornos mentais menores acometem cerca de 30\% dos trabalhadores ocupados; os transtornos mentais graves, cerca de 5 a 10\%. No Brasil, dados do INSS sobre a concessão de aposentadoria por invalidez, por incapacidade definitiva para o trabalho, mostram que os transtornos mentais ocupam o terceiro lugar entre as causas dessas ocorrências.

Dados mais recentes do Ministério da Previdência Social (BRASIL, 2012) dão conta de que os transtornos mentais e comportamentais ocupam o terceiro lugar em quantidade de concessões de auxílio-doença acidentários e não vêm acompanhando a tendência de queda no número de acidentes de trabalho no Brasil. Pelo contrário, de 2008 para 2009, o número de afastamentos do trabalho em decorrência de transtornos mentais e comportamentais subiu de 12.818 para 13.478. Em 2010, esse número teve uma queda, passando para 12.150. No entanto, voltou a subir em 2011, passando para 12.337. Entre os transtornos mentais e comportamentais, as doenças que mais afastaram os trabalhadores em 2011 foram Episódios Depressivos, Outros Transtornos Ansiosos e Reações ao Estresse Grave e Transtornos de Adaptação. Note-se que esses dados evidenciam uma condição (sofrimento psíquico) cujo estabelecimento do nexo causal reveste-se de extrema complexidade e representa um dos grandes desafios que se apresenta ao campo psi (CFP, 2010), especialmente considerando-se o que apontam Seligmann-Silva et al. (2010b, p. 187), "os aspectos sociais, econômicos e organizacionais, assim como os processos psicossociais em suas repercussões sobre a subjetividade do trabalhador, são minimizados ou ignorados". Os autores, com base em diversas pesquisas, acrescentam que

adicionalmente à relevância estatística, há que se considerar que o agravamento dos transtornos mentais dos trabalhadores submetidos a condições penosas é frequente, levando-os ao consumo de drogas, a sofrerem acidentes de trabalho, à incapacidade para o trabalho, ao afastamento do trabalho por tempo prolongado e à exclusão do mercado de trabalho, resultando em grandes custos ao Estado e à sociedade (SELIGMANN-SILVA et al. 2010b, p. 188-189).

Conforme o Ministério da Saúde (BRASIL, 2001), a contribuição do trabalho para as alterações da saúde mental das pessoas dá-se a partir de ampla gama de aspectos: desde fatores pontuais, como a exposição a determinado agente tóxico, até a complexa articulação de fatores relativos à organização do trabalho, como a divisão e parcelamento das tarefas, as políticas de gerenciamento das pessoas e a estrutura hierárquica organizacional. 
Os transtornos mentais e do comportamento relacionados ao trabalho resultam, assim, não de fatores isolados, mas de contextos de trabalho em interação com o corpo e o aparato psíquico dos trabalhadores.

As ações implicadas no ato de trabalhar podem atingir o corpo dos trabalhadores, produzindo disfunções e lesões biológicas, mas também reações psíquicas às situações patogênicas de trabalho, além de poderem desencadear processos psicopatológicos especificamente relacionados às condições do trabalho desempenhado pelo trabalhador.

O trabalho ocupa um lugar fundamental na dinâmica do investimento afetivo das pessoas (DEJOURS, 1993). Condições favoráveis à livre utilização das habilidades dos trabalhadores, da inteligência da prática (DEJOURS; ABDOUCHELI; JAYET, 1994; DEJOURS, 1995) e que possibilitem aos trabalhadores um nível significativo de controle sobre seu processo de trabalho têm sido identificadas como importantes requisitos para que a atividade de trabalho possa proporcionar prazer, bem-estar e saúde, deixando de provocar doenças. Por outro lado, o trabalho desprovido de significação, sem suporte social, não reconhecido ou que se constitua em fonte de ameaça à integridade física e/ou psíquica, pode desencadear sofrimento psíquico.

\section{Desafios para a Psicologia}

Segundo consta no documento elaborado pelo Centro de Referência Técnica em Psicologia e Políticas Públicas (CREPOP, 2008), o psicólogo poderá deparar-se com as questões do processo saúde-doença em sua relação com o trabalho, independentemente do lugar em que esteja atuando. Contudo, compreender as especificidades da relação trabalho e saúde requer um pensar e fazer diferenciados, que remetem à consideração dos aspectos da organização, processo e condições de trabalho, a compreensão da vivência subjetiva no trabalho e as repercussões para a saúde mental dos trabalhadores. Dessa forma, é preciso reconhecer a subjetividade no trabalho, o significado que as pessoas atribuem a determinadas situações, o modo como cada um reage a partir da sua história de vida, de seus valores, das suas crenças, das suas experiências e das suas representações sobre a atividade desenvolvida.

A atuação do psicólogo, no que tange aos transtornos mentais e do comportamento relacionados ao trabalho não pode ficar restrita a uma unidade especializada. Ela deve ocorrer nos mais diversos serviços, tanto os privados quanto aqueles da rede SUS (Sistema Único de Saúde), tais como unidades de atenção básica, ambulatórios de especialidades, Centros de Atenção Psicossocial (CAPS), hospitais e serviços de vigilância em saúde.

O psicólogo, na sua prática clínica, não deve perder de vista a centralidade do trabalho na compreensão da subjetividade humana. Essa dimensão deverá necessariamente ser levada em conta. Contudo, deve-se ressaltar que é na abordagem interdisciplinar que se pode dar conta da amplitude dos pro- 
blemas de saúde relacionados ao trabalho, o que contempla mais amplamente a orientação e recomendação de medidas que contribuam para prevenção, diagnóstico, tratamento e reabilitação.

As terapêuticas desenvolvidas pelos psicólogos podem ser voltadas para indivíduos ou grupos de trabalhadores. Destaque especial vem sendo atribuído às atividades grupais com portadores de doenças crônicas. Em tais atividades, são adotadas diversas perspectivas teóricas. De modo geral, os grupos têm caráter informativo-terapêutico, valorizam o conhecimento e a subjetividade dos trabalhadores e visam à ressignificação do processo de adoecimento, além de legitimar o seu discurso, estimular a sua participação e autonomia em relação ao tratamento, o que propicia o autoconhecimento.

Jacques (2003) discorre sobre algumas das principais abordagens no âmbito da saúde/doença mental e trabalho e suas interseções com a Psicologia e, particularmente, com a Psicologia Social. Identifica, no conjunto das "teorias sobre estresse", uma ênfase nos pressupostos cognitivos comportamentais, na metodologia quantitativa e uma aproximação com os postulados da "Psicologia Social científica"; reconhece na "Psicodinâmica do Trabalho", fundamentos psicanalíticos na concepção teórica, conceitual, de ciência e pesquisa. Distingue, nos estudos e pesquisas com base no "modelo epidemiológico e/ou diagnóstico" e nas pesquisas em "subjetividade e trabalho", pressupostos compartilhados pela "Psicologia Social histórico-crítica", com prevalência para o diagnóstico psicopatológico ou para as experiências e vivências dos trabalhadores.

Diante dos desafios e da complexidade da questão da saúde mental no trabalho diversas pesquisas e publicações vêm sendo produzidas no Brasil. As temáticas são diversificadas, englobando estudos e pesquisas empíricas acerca de estresse, burnout, assédio moral, relação desemprego prolongado e saúde/adoecimento psicossocial, reabilitação profissional, relações de gênero, epidemiologia em saúde mental e trabalho, sentido do trabalho, discurso e prática corporativa, etc. Os grupos pesquisados variam, havendo um grande número de estudos envolvendo profissionais de educação e saúde, petroleiros, bancários e profissionais da construção civil. Tão variados quanto os sujeitos pesquisados são as teorias, métodos e técnicas empregados para abarcar as suas singularidades e dos diferentes mundos do trabalho existentes.

Silva Filho e Jardim (1997) organizaram uma coletânea de ensaios que abordam as áreas temáticas Trabalho \& Subjetividade, Trabalho \& Doença Mental, Trabalho \& Gênero e Trabalho \& Profissionais de Saúde.

Saúde Mental \& Trabalho também foi o tema das "Leituras" organizadas por Jacques e Codo (2002).

Um dossiê específico sobre o tema, intitulado "O mundo contemporâneo do trabalho e a saúde mental do trabalhador", foi publicado em dois volumes pela Revista Brasileira de Saúde Ocupacional (2010; 2011), reunindo diversos e importantes trabalhos. 
Glina e Rocha (2010), por sua vez, organizaram um livro reunindo autores de diferentes abordagens, cuja proposta foi percorrer um caminho da teoria à prática em saúde mental no trabalho. Para tal empreitada, traçaram os seguintes objetivos: apresentar conceitos básicos e metodológicos que permitam compreender as dinâmicas de trabalho debilitantes, as vivências subjetivas e os mecanismos de enfrentamento e defesa adotados individual e coletivamente pelos trabalhadores. Adicionalmente, propuseram-se apresentar, através dos diversos autores, formas de reconhecer os agravos à saúde mental possivelmente relacionadas à situação de trabalho; destacar técnicas que auxiliem no estabelecimento do nexo causal com o trabalho e avaliação da incapacidade, bem como na elaboração de laudos técnicos e relatórios; discutir casos reais ilustrativos das diversas abordagens; e analisar diferentes níveis de prevenção em saúde mental no trabalho.

Já, Bendassolli e Soboll (2011) ocuparam-se de abordar as "clínicas do trabalho", um conjunto de teorias que têm como foco de estudo a relação entre trabalho e subjetividade. Estão aí compreendidas a "Clínica da Atividade", a "Psicossociologia", a "Psicodinâmica do Trabalho" e a "Ergologia". "Apresentando uma diversidade epistemológica, teórica e metodológica, o objeto comum dessas teorias é a situação do trabalho, que, em síntese, compreende a relação entre o sujeito, de um lado, e o trabalho e o meio, de outro". (BENDASSOLLI; SOBOLL, p. 3, grifo dos autores). Ressalte-se que muitos autores identificam no Modelo Operário Italiano de luta pela saúde (ODDONE et al., 1986[1977]; ODDONE; RE; BRIANTI, 1977) uma importante colaboração para a emergência do que hoje se denomina "clínica do trabalho" (ao lado das experimentações de Tosquelles, Le Guillant e outros, na França).

Não obstante todo esse referencial teórico-metodológico-técnico disponível (materiais imprescindíveis para quem quer se informar e se atualizar sobre o tema), lembramos que o grande desafio que se apresenta ao psicólogo consiste no estabelecimento do nexo causal entre os transtornos mentais e os aspectos organizacionais do trabalho. De acordo com Silva et al. (2009), embora os transtornos mentais estejam entre as principais causas de perdas de dias no trabalho, o conhecimento produzido, principalmente no Brasil, ainda é insuficiente e inconclusivo para uma compreensão mais aprofundada dessa problemática. Os autores confirmam que uma das principais dificuldades é o estabelecimento da relação de causalidade entre os transtornos mentais e do comportamento ao trabalho e vão além, apontando outra dificuldade, a identificação das situações laborais que favorecem o aparecimento e o agravamento dessas doenças.

Podemos citar ainda outros fatores que contribuem para aumentar ainda mais essa dificuldade na vinculação do quadro clínico de transtorno mental com o trabalho/atividade desempenhada: 1) a inexistência de uma lesão física evidenciável (exceto no caso de um traumatismo crânio-encefálico, por exemplo); 2) a desqualificação das manifestações de sofrimento que não coincidem com as referências diagnósticas dos manuais. Geralmente deparamo-nos com um quadro representado por uma síndrome inespecífica, um sofrimento difuso, que inclui mal-estar, medo, ansiedade, depressão, nervosismo, tensão, fadiga, perda de apetite, distúrbios do sono e psicossomáticos (gastrites, crises hipertensivas, etc.); 3) o não reconheci- 
mento social de que alguns casos de sofrimento psíquico podem comprometer a capacidade de trabalhar (ausência de consenso e de definição na CID-10 e a existência de uma lista oficial que reconhece, no âmbito dos ministérios da Saúde e da Previdência Social, alguns transtornos mentais e comportamentais relacionados ao trabalho, mas que permanece ainda restrita; 4) o fato de que o processo de adoecimento é específico para cada pessoa (história de vida e de trabalho), levando, muitas das vezes à individualização de um problema de caráter coletivo.

Nesse sentido, o documento do Centro de Referência Técnica em Psicologia e Políticas Públicas (CREPOP, 2008), anteriormente citado, orienta que a investigação diagnóstica em saúde mental e trabalho deve combinar diferentes técnicas, como, por exemplo, entrevistas e testes psicológicos. E prossegue afirmando que no processo de investigação diagnóstica, deve-se sempre perguntar sobre o trabalho, realizar uma anamnese ocupacional, levantar aspectos da organização do trabalho, identificar as exigências físicas e mentais, inquirir sobre a percepção do trabalhador a respeito dos riscos, obter informações sobre o posto de trabalho, as condições ambientais e o processo de trabalho. Observa-se, assim, que o nexo entre saúde/doença mental exige olhar e atuação interdisciplinar, na qual o psicólogo tem papel de destaque.

É importante que, ao buscar estabelecer nexo entre trabalho e saúde/doença mental, sejam considerados o contexto laboral, a subjetividade do trabalhador e, principalmente, a relação entre esses dois aspectos.

\section{CONSIDERAÇÕes FINAIS}

É importante lembrar que o olhar para as questões que envolvem a saúde do trabalhador deve ser incorporado pelo psicólogo independentemente do tipo de serviço no qual esteja inserido ou de sua área de atuação.No Brasil, contudo, verifica-se que pouca ênfase tem sido dada à categoria trabalho no contexto geral da formação do psicólogo. Ocorre também na maior parte dos atendimentos clínicos (públicos ou privados), na rede básica ou em unidades de emergência, além de na atuação nas empresas.

É muito importante que, ao atender um indivíduo, o profissional esteja atento à possibilidade de que suas queixas estejam relacionadas ao trabalho. Tradicionalmente, a formação do psicólogo não contempla a relação entre trabalho e saúde mental, e muitos profissionais acabam por negligenciá-la. A relação traba1ho/subjetividade ainda não foi devidamente reconhecida nesse contexto, o que representa uma séria barreira para a atuação desse profissional em todas as áreas, sobretudo no campo da Saúde do Trabalhador. Daí infere-se que é importante que os profissionais com experiência nessa área, que vêm lidando com os transtornos mentais e do comportamento relacionados ao trabalho de forma eficaz, coloquem seus conhecimentos à disposição nos mais diversos campos de atuação da psicologia, cobrindo uma lacuna ainda existente na área e interagindo com os outros psicólogos e também com profissionais de outras especialidades afins. 


\section{REFERÊNCIAS}

BENDASSOlLI, P. F.; SOBOLL, L. A. P. (Org.). Clínicas do Trabalho. São Paulo: Atlas, 2011.

BRASIL. Presidência da República. Decreto $n^{\circ} 3.048$, de 6 de maio de 1999. 1999a. Disponível em: < http://www.planalto.gov.br/ccivil_03/decreto/d3048. htm>. Acesso em: 26 maio 2010.

BRASIL. Ministério da Saúde. Portaria $n^{\circ}$ 1339/GM. Em 18 de novembro de 1999. 1999b. Disponível em: <http://dtr2001.saude.gov.br/sas/PORTARIAS/ Port99/GM/GM-1339.html>. Acesso em: 18 jun. 2010.

BRASIL. Ministério da Saúde. Doenças relacionadas ao trabalho: manual de procedimentos para os serviços de saúde. Brasília: Ministério da Saúde do Brasil, 2001. Disponível em: <http://dtr2001.saude.gov.br/editora/produtos/livros/ pdf/02_0388_M1.pdf>. Acesso em: 15 abr. 2010.

BRASIL. Ministério da Previdência e Assistência Social. Previdência em questão. Informativo Eletrônico, no 59, de 16 a 29 de fevereiro de 2012. Disponível em $<$ http://www.mps.gov.br/arquivos/office/4_120326-105114-231.pdf>. Acesso em 26 out. 2012.

CENTRO DE REFERÊNCIA TÉCNICA EM PSICOLOGIA E POLÍTICAS PÚBLICAS (CREPOP). Saúde do Trabalhador no âmbito da Saúde Pública: referências para a atuação do(a) psicólogo(a). Brasília: CFP, 2008.

CONSELHO FEDERAL DE PSICOLOGIA (CFP). Nexo causal: desafio para o campo da saúde do trabalhador. Jornal do Federal, Brasília, Ano XXII, n. 96, p. 10-11, mar. 2010. Disponível em: <http://site.cfp.org.br/wp-content/ uploads/2012/03/jornal_federal_96.pdf>. Acesso em: 22 jun. 2010.

DEJOURS, C. Addendum theórique: de la psychopathologie a la psychodynamique du travail. In . Travail, usure mentale: essai de psychopathologie du travail. Nouvelle édition augmenté. Paris: Bayard, 1993. p. 204-253.

DEJOURS, C. Inteligência operária e organização do trabalho: a propósito do modelo japonês de produção. In: HIRATA, H. (Org.) Sobre o modelo japonês. São Paulo: Edusp, 1995. p. 281-309.

DEJOURS, C.; ABDOUCHELI, E.; JAYET, C. Psicodinâmica do Trabalho: contribuições da escola dejouriana à análise da relação prazer, sofrimento e trabalho. São Paulo: Atlas, 1994. 
GLINA, D. M. R.; ROCHA, L. E. Saúde mental no trabalho: da teoria à prática. São Paulo: Roca, 2010.

JACQUES, M. G. C. Abordagens teórico-metodológicas em saúde/doença mental \& trabalho. Psicologia \& Sociedade, [S.1.], v. 15, n. 1, p. 97-116, jan/jun. 2003.

JACQUES, M. G. C., CODO, W. (Org.). Saúde mental \& trabalho: leituras. Petrópolis, RJ: Vozes, 2002.

ODDONE, I. et al. Ambiente do trabalho: a luta dos trabalhadores pela saúde (1977). São Paulo: Hucitec, 1986.

ODDONE, I.; RE, A.; BRIANTI, G. Esperienza operaia, coscienza di classe e psicologia del lavoro. Turim: Giulio Einaudi, 1977.

REVISTA BRASILEIRA DE SAÚDE OCUPACIONAL. São Paulo: Fundação Jorge Duprat Figueiredo de Segurança e Medicina do Trabalho FUNDACENTRO, v. 35, n. 122, jul/dez. 2010.

REVISTA BRASILEIRA DE SAÚDE OCUPACIONAL. São Paulo: Fundação Jorge Duprat Figueiredo de Segurança e Medicina do Trabalho FUNDACENTRO, v. 36, n. 123, jan/jun. 2011.

SELIGMANN-SILVA, E. et al. Saúde do Trabalhador no início do século XXI (Editorial). Rev. bras. Saúde ocup., São Paulo, v. 35, n. 122, p. 185-186, 2010a.

SELIGMANN-SILVA, E. et al. O mundo contemporâneo do trabalho e a saúde mental do trabalhador (Apresentação). Rev. bras. Saúde ocup., São Paulo, v. 35, n. 122, p. 187-191, 2010 b.

SILVA FILHO, J. F.; JARDIM, S. (Org.). A danação do Trabalho: relações de trabalho e o sofrimento. Rio de Janeiro: Te Corá, 1997.

SILVA, G. G. J. et al. Considerações sobre o transtorno depressivo no trabalho. Revista Brasileira de Saúde Ocupacional, São Paulo, v. 34, n. 119, p. 79-87, jan./ jun. 2009.

Recebido em: 11 de julho de 2010 Aceito em: 07 de novembro de 2012 
